DOI: $10.21105 /$ joss. 02174

\section{Software}

- Review ¿

- Repository ¿

- Archive cto

Editor: Yuan Tang ¿

\section{Reviewers:}

- Osara-02

- Qejhigson

Submitted: 02 March 2020 Published: 05 August 2020

\section{License}

Authors of papers retain copyright and release the work under a Creative Commons Attribution 4.0 International License (CC BY 4.0).

\title{
Surprise: A Python library for recommender systems
}

\section{Nicolas Hug ${ }^{1}$}

1 Columbia University, Data Science Institute, New York City, New York, United States of America

\section{Summary}

Recommender systems aim at providing users with a list of recommendations of items that a service offers. For example, a video streaming service will typically rely on a recommender system to propose a personalized list of movies or series to each of its users. A typical problem in recommendation is that of rating prediction: given an incomplete dataset of useritem interations which take the form of numerical ratings (e.g. on a scale from 1 to 5), the goal is to predict the missing ratings for all remaining user-item pairs.

Surprise is a Python library for building and analyzing rating prediction algorithms. It was designed to closely follow the scikit-learn API (Buitinck et al., 2013; Pedregosa et al., 2011), which should be familiar to users acquainted with the Python machine learning ecosystem.

Surprise provides a collection of estimators (or prediction algorithms) for rating prediction. Among others, classical algorithms are implemented such as the main similarity-based algorithms (Aggarwal \& others, 2016), as well as algorithms based on matrix factorization like SVD (Koren, Bell, \& Volinsky, 2009) or NMF (Lee \& Seung, 2001). It also supports tools for model evaluation like cross-validation iterators and built-in metrics à la scikit-learn, as well as tools for model selection and automatic hyper-parameter search, namely grid search and randomized search. Thanks to simple primitives and a light API, users can also implement their own recommendation technique with a minimal amount of code.

Classical datasets such as the MovieLens datasets (Harper \& Konstan, 2015) are directly available in the package, but user-defined datasets are also supported either by loading csv files, or by using pandas dataframes (McKinney, 2010).

Surprise is mainly written in Python, while the computationally intensive parts are optimized with Cython (Behnel et al., 2011). Internally, Surprise relies on built-in Python data structures (mainly dictionaries) as well as numpy arrays (Walt, Colbert, \& Varoquaux, 2011).

Surprise was designed to be useful to researchers who want to quickly explore new recommendation ideas by supporting the creation of custom prediction algorithms, but can also serve as a learning resource for students and less experienced users thanks to its detailed documentation.

Other popular recommendation libraries with similar functionalities include LibRec (Guo, Zhang, Sun, \& Yorke-Smith, n.d.) (Java) or MyMediaLite (Gantner, Rendle, Freudenthaler, \& Schmidt-Thieme, 2011) (C\#). In Python, OpenRec (Yang, Bagdasaryan, Gruenstein, Hsieh, \& Estrin, 2018) and Spotlight (Kula, 2017) support neural-network inspired algorithms; implicit ${ }^{1}$ is specialized in implicit feedback recommendation, and LightFM (Kula, 2015) implements a hybrid algorithm based on matrix factorization. To the best of our knowledge, Surprise is the only library to provide a scikit-learn like API with model selection tools, and with a focus on explicit rating prediction.

\footnotetext{
${ }^{1}$ https://github.com/benfred/implicit
} 


\section{Example}

Here is a simple example showing how to (down)load a dataset, split it into five folds for cross-validation, and compute the Mean Average Error (MAE) and the Root Mean Squared Error (RMSE) of the SVD algorithm.

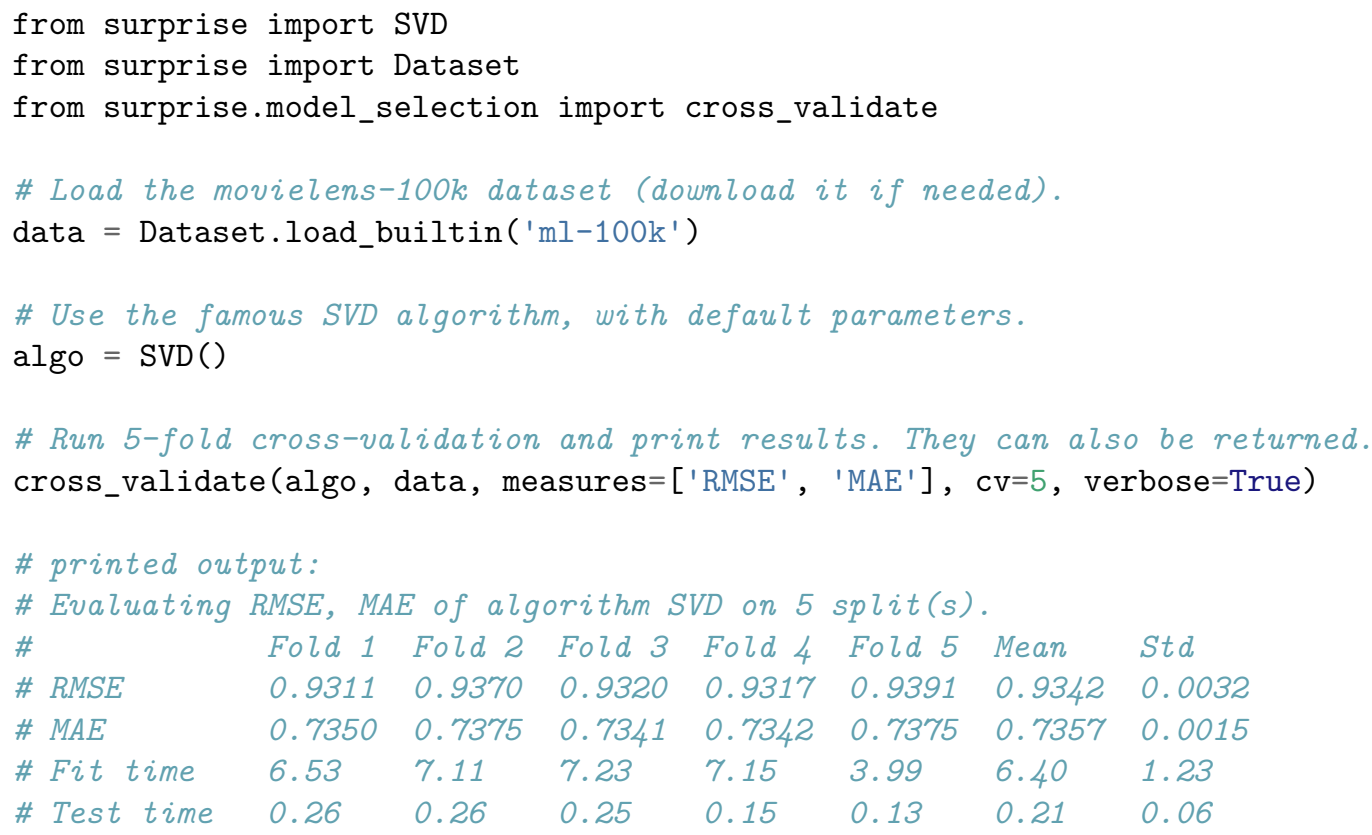

\section{Acknowledgements}

We are grateful to all the people who have contributed to the software, with special thanks to Maher Malaeb and David Stevens for the hyper-parameter searches, and to Lauriane Ducasse for the logo design.

\section{References}

Aggarwal, C. C., \& others. (2016). Recommender systems (Vol. 1). Springer. doi:10.1007/ 978-3-319-29659-3

Behnel, S., Bradshaw, R., Citro, C., Dalcin, L., Seljebotn, D. S., \& Smith, K. (2011). Cython: The best of both worlds. Computing in Science \& Engineering, 13(2), 31-39. doi:10.1109/ MCSE.2010.118

Buitinck, L., Louppe, G., Blondel, M., Pedregosa, F., Mueller, A., Grisel, O., Niculae, V., et al. (2013). API design for machine learning software: Experiences from the scikit-learn project. In ECML PKDD Workshop: Languages for Data Mining and Machine Learning (pp. 108-122).

Gantner, Z., Rendle, S., Freudenthaler, C., \& Schmidt-Thieme, L. (2011). MyMediaLite: A Free Recommender System Library. In Proceedings of the 5th ACM Conference on Recommender Systems (RecSys 2011).

Guo, G., Zhang, J., Sun, Z., \& Yorke-Smith, N. (n.d.). LibRec: A Java Library for Recommender Systems. 
Harper, F. M., \& Konstan, J. A. (2015). The Movielens Datasets: History and Context. ACM Transactions on Interactive Intelligent Systems (TIIS), 5(4), 1-19.

Koren, Y., Bell, R., \& Volinsky, C. (2009). Matrix factorization techniques for recommender systems. Computer, 42(8), 30-37.

Kula, M. (2015). Metadata Embeddings for User and Item Cold-start Recommendations. In T. Bogers \& M. Koolen (Eds.), Proceedings of the 2nd Workshop on New Trends on Content-Based Recommender Systems co-located with 9th ACM Conference on Recommender Systems (RecSys 2015), Vienna, Austria, September 16-20, 2015., CEUR workshop proceedings (Vol. 1448, pp. 14-21). CEUR-WS.org. Retrieved from http: //ceur-ws.org/Vol-1448/paper4.pdf

Kula, M. (2017). Spotlight. https://github.com/maciejkula/spotlight; GitHub.

Lee, D. D., \& Seung, H. S. (2001). Algorithms for non-negative matrix factorization. In Advances in Neural Information Processing Systems (pp. 556-562).

McKinney, W. (2010). Data Structures for Statistical Computing in Python. In S. van der Walt \& J. Millman (Eds.), Proceedings of the 9th Python in Science Conference (pp. 51-56). doi:10.25080/Majora-92bf1922-00a

Pedregosa, F., Varoquaux, G., Gramfort, A., Michel, V., Thirion, B., Grisel, O., Blondel, M., et al. (2011). Scikit-learn: Machine Learning in Python. Journal of Machine Learning Research, 12, 2825-2830.

Walt, S. van der, Colbert, S. C., \& Varoquaux, G. (2011). The numpy array: A structure for efficient numerical computation. Computing in Science \& Engineering, 13(2), 22-30. doi:10.1109/MCSE.2011.37

Yang, L., Bagdasaryan, E., Gruenstein, J., Hsieh, C.-K., \& Estrin, D. (2018). OpenRec: A Modular Framework for Extensible and Adaptable Recommendation Algorithms. In Proceedings of the Eleventh ACM International Conference on Web Search and Data Mining, WSDM '18 (pp. 664-672). New York, NY, USA: Association for Computing Machinery. doi:10.1145/3159652.3159681 